\title{
TRATAMENTO DE PARASITOSES INTESTINAIS PELA ASSOCIAÇÃO PIPERAZINA-TIABENDAZOL *
}

\author{
Adelina S. Velho Soli,** Hugo Americano do Brasil, *** \\ Léa Camillo-Coura $* * * *$ e J. Rodrigues Coura $* * * * *$
}

\begin{abstract}
Os autores apresentam os resultados obtidos com o emprego da associação antihelmintica piperazina-tiabendazol no tratamento de infecção por Ascaris Iumbricoides $e$ ancilostomídeos. Considerando a cura de $81 \%$ dos casos de ascaridiase e de $80 \%$ dos casos de ancilostomiase, bem como a ótima tolerância ao medicamento, acreditam que esta associação medicamentosa possa ser empregada com sucesso para o tratamento das referidas parasitoses.
\end{abstract}

A associação parasitária intestinal é ..ma constante na patologia do homem bra::.eiro, especialmente daquele que vive em zona rural ou mesmo nas regióes peri-ur canas; são frequentes as infecções por $\therefore$ scaris lumbricoides, Trichuris trichiura, incilostomideos, Enterobius vermicularis e ¡ outros helmintos. Um grande número de medicamentos tem sido empregado para o ¿Eu tratamento, sendo desejável o emprego ¿e um antihelmíntico que tenha um amplo ¡spectro de ação.

Para o tratamento da ascaríase, reco: hecidamente a piperazina, o tetramisole E o pamoato de pirantel podem ser empregados com resultados bastantes satiscatórios $(1,2,3,4,12)$; a ancilostomíase responde ao tratamento com o tetracloroe-ileno, com o befênio, com a fenilenodiiso- tiocianato e com o tiabendazol, como demonstram as experiências de vários investigaciores $(1,2,3,4,5,6)$

A enterobíase é sensivel ao tratamento com a piperazina, o pirvínio e o tiabendazol. O Trichuris trichiura, no entanto, mostra-se um helminto resistente aos diferentes antihelminticos ensaiados (3).

A associacāo piperazina-tiabendazol tem-se mostrado de valor no tratamento de poliparasitoses intestinais $(10,11)$ proporcionando significativos percentuais de cura, motivo pelo qual resolvemos ensaiá-la em nosso Serviço.

\section{MATERIAL E MÉTODOS}

Foram relacionados 111 individuos apresentando a associaçāo Ascaris-ancilostomideos, em 101 destes associando-se tam-

\footnotetext{
* Trabalho do Departamento de Medicina Preventiva e Social da Faculdade de Medleina da U.F.R.J e da SUCAM, Ministério da Saúde, realizado com o auxilio da COPERTIDE.

* Auxiliar de Ensino

** Chefe da Circunscrição Jacarepagua, SUCAM, M.S.

$* * *$ Professor Adjunto

**** Professor Titular, Chefe do Departamento.

Recebido pela publicação em 5.4.1972.
} 
bém o Trichuris trichiura. Todos eram provenientes dos Ambulatórios da Clínica de Doenças Infectuosas e Parasitárias da Faculdade de Medicina da U.F.R.J. e da Circunscrição Jacarepaguá, SUCAM, Ministério da Saúde. 61 pacientes eram do sexo masculino e 50 do sexo feminino; 59 brancos, 41 pardos e 11 pretos; 64 tinham de 2 a 14 anos, a idade máxima no grupo submetido a estudo sendo de 51 anos.

o diagnóstico parasitológico foi feito através do método de Hoffman-Pons-Janer em todos os pacientes, complementado pela contagem de ovos pelo método de Stoll; o contrôle de cura foi realizado com as mesmas técnicas, no mínimo em duas oportunidades, durante as três primeiras semanas após o tratamento. Não foram consiceradas as técnicas indicadas para diagnóstico do estrongilcidiase e da enterobíase, pelo que não se inclui o estudo do medicamento sobre estes parasitos no presente trabalho.

O medicamento usado * foi administrado na dose de $5 \mathrm{ml}$ para cacia $15 \mathrm{~kg}$ de peso corporal, dose esta fracionacia em duas tomadas ao dia, e repetida durante 2 dias consecutivos. Cada $10 \mathrm{ml}$ do medicamento, que é apresentado em suspensão, contém $750 \mathrm{mg}$ de hexahidrato de piperazina e 375 $\mathrm{mg}$ de tiabendazol.

Foram consideracias a eliminação de parasitos após a ingestão do medicamento e eventuais manifestaçōes de intolerância que se pudessem observar.

\section{RESULTADOS}

No que se refere a Ascaris lumbricoides, 90 dos 111 casos tratados apresentaram cura parasitológica aos exames de controle, evidenciando $81 \%$ de curas; dos portadores de ancilostomiase, 89 apresentaramse curacios, correspondendo a $80 \%$ de negativação aos exames parasitológicos. 49 dos 101 pacientes de infecção por Trichuris trichiura também tiveram seus exames coprológicos negativos, correspondendo a $48 \%$ de curas.

Os resultados podem ser observados no Quadro I.

Em 57 casos observou-se a eliminação de número variável de exemplares de $A$ scaris lumbricoides. Em 15 casos ( $13 \%$ ) foram referidas manifestações de intolerância do tipo de tonturas, cefaléia, náuseas, dor abdominal, vômitos e obstipação intestinal (em um caso, perdurando por 5 dias), todos eles, no entanto, de pequena monta.

\section{COMENTÁRIOS}

Os resultados obticios na presente investigação são concordantes com os de outros pesquisadores que ensaiaram a mesma associação medicamentosa, como Meira e Elkis e Ferrioli Filho e cols. $(10,11)$

Aigumas consideraçōes devem sc $\mathrm{r}$ feitas, no entanto, em relação aos indices de cura

\section{QUADRO I \\ PACIENTES SUBMETIDOS AO TRATAMENTO COM A ASSOCIAÇÃO PIPERAZINA-TIABENDAZOL}

\begin{tabular}{|c|c|c|c|c|}
\hline TIPO DE PARASITO & $\begin{array}{l}\text { Número de } \\
\text { Pacientes } \\
\text { Tratados }\end{array}$ & $\begin{array}{c}\text { Carga Média } \\
\text { Parasitária } \\
\text { Antes Trata. } \\
\text { (ovos por g. de } \\
\text { fezes) }\end{array}$ & $\begin{array}{c}\text { No Pacientes } \\
\text { Curados }\end{array}$ & Cura \\
\hline Ascaris lumbricoides & 111 & 696 & 90 & 81 \\
\hline Ancilostomídeos & 111 & 1341 & 89 & 80 \\
\hline Trichuris trichiura & 101 & 737 & 49 & 48 \\
\hline
\end{tabular}

* Gamaverm (Labofarma S.A.). 
obtidos no tratamento da ancilostomíase; em trabalho anterior um de nós, (3) empregando o tiabendazol na dose de 25 $\mathrm{mg} / \mathrm{kg}$ cie peso ao dia, pelo período de 3 dias, obteve $63 \%$ de curas $\mathrm{\epsilon m} 39$ casos tratados; no presente estudo, a cura obtida foi de $\mathbf{8 0} \%$ dos cascs, embora com doses menores do tiabendazol durante 2 dias consecutivos; em ambos os casos a carga parasitária foi baixa nos grupos estudados, com uma média de 520 ovos cie ancilostomídeos por exame de fezes para o primeiro e de 696 no atual estudo; os indices de cura agora obtidos vêm confirmar os trabalhos iniciais com relação à eficácia do tiabendazol no tratamento da ancilostomíase.

No tocante à ascaridíase são também bastante satisfatórios os percentuais de cura obtidos, principalmente se considerarmos o fato de que a asscciação medicamentosa é significativamente eficaz para o tratamento ciesta parasitose em associação à ancilostomíase, sem promover as manifestações de intolerância que comumente se observam quando do emprego isolado da maioria dos ancilostomicidas, permitin- do um apreciável número de curas da associação Ascaris-ancilostomideos.

Os resultados em relação ao parasitismo do Trichuris trichiura deixam ainda a desejar, como ocorre com os diferentes medicamentos ou associações medicamentosas empregadas para o seu tratamento. Infelizmente não nos foi possivel avaliar a eficácia sobre o S.stercoralis, acreditando, no entanto, baseados nas experiências anteriores com o uso do tiabendazol isoladamente em diferentes esquemas terapêuticos e mesmo em associação à piperazina $(3,7,8,9,10)$ que os resultados sejam promissores. O estudo da eficácia da associação piperazina-tiabendazol sobre o E. vermicularis está sendo levado a efeito em nosso Serviço e os resultados serão publicados oportunamente.

Finalizancio, consideramos que a associação medicamentosa em apreço possa ser empregada, dadas a sua eficácia e ótima tolerância, para o tratamento de duas das parasitoses intestinais mais frequentes em nosso meio, quais sejam Ascaris e ancilostomideos.

The authors tried piperazine plus thiabendazole for the treatment of Ascaris lumbricoides and hookworn infections. The arug was given jor 2 consecutive days in a daily dose of $750 \mathrm{mg}$ of piperazine hydrate and $375 \mathrm{mg}$ of thiabendazole for $33 \mathrm{~kg}$ of body weight. Cure was obtained in $81 \%$ of those infected with A. lumbricoicies and in $80 \%$ of hookworm infections. Tolerance to the drug was considereà very good.

\section{REFERENCIAS BIBLIOGRÁFICAS}

1 - AMATO NETO, V.; LEVI G.; CAMPOS, R, CORREA, M.O.A. ; FLEURY \& CAMPOS, L.L. - Observaçōes sobre a atividade antihelmíntica do pamoato de pirantel. Rev. Soc. Bras. Med. Trop.; 3: 19, 1969.

2 - CAMILLO-COURA, LEA, WARD, J.; FLORÊNCIO, C.; CARVALHO, H.T.; FERREIRA, L.F. \& RODRIGUES dá SILVA, J. - Ensaios sobre terapêu- tica antihelmintica $\mathrm{ccm}$ sais de piperazina, triclorcfenol piperazina, tetracloreotileno e hidroxinaftoato $d \epsilon$ befênio. Anais do XI Congresso $\mathrm{Na}$ cional de Medicina, Rio, 1962.

3 - CAMILLO-COURA, LîA - Contribuição ao estudo das Geohelmintiases Tese de Docência a Faculdade de Medicina da U.F.R.J..; 1970, 215 pgs. 
4 - CAMILLO-COURA, LÉA, VELHO SOLI, A.; AMERICANO DO BRASIL, $\mathrm{H}$. \& RODRIGUFS COURA, J. - Tratamento da ancilostomíase e de outros helmintos intestinais pelo pamoato de pirantel. Em publicação.

5 - CAMPOS, R. - Acão antihelmíntica do hidroxinaftoato de befênio. Tese. Faculdade de Medicina da Univ. de S. Paulo, 1960 .

6 - CAMPOS, R.; AMATO NETO, V.; ZERATI, A.; GOMES, M.C.O. \& ALBUQUERQUE, F.J.M. - Tratamento da ancilostomiase mediante o emprêgo de ciiversos compostos. Proc. VIIth Int. Cong. Trop. Med. \& Mal.; 2: 180, 1963.

7 - CROCE, J.; CAMPOS, R.; AMATO NETO, V.; PIRES, C.D.; CASTRO, A.C.M.; LAURENTI, R. \& ALBUQUERQUE, F.J.M. - Tratamento da estrongiloidose pelo tiabendazol. Rev. Inst. Med. S. Paulo, 5: 194-197, 1963.

8 - FERRAZ, W. G.; CABECA, M.; SARRA, C. \& GUIMARÃES, S. S. - Trata- mento da estrongiloidíase e da ascaridíase com Thiabendazol. Hospital, 64: 109-116, 1963.

9 - FRANK, K.H. - Clinical trials with thiabenciazole against human strongyloidiasis. Am. J. Trop. Med. Hyg.; 12: 211-214, 1963.

10 - FERRIOLI FILHO, F.; DUARTE, G. G.; TEPUEL, J.R. \& FRANCO, A.R. Fstudos de uma associacão antihelmíntica em parasitoses intestinais multiplas. Rev. Soc. Bras. Med. Trop.; 5: 209, 1971.

11 -MEIRA, D. A. \& ELKIS, H. - Parasitoses intestinais multiplas em aciultos. Tratamento pela associação tiabendazol e piperazina. Hospital, 73 : $265,1868$.

12 - SWATZWELDER, C.; MILLER, J.N. \& SAPPENFIELD, R.W. - The effective use of piperazine for the treatment of human helmintiasis. Gastroenterology, 33: 87, 1957. 